\title{
Conflitos bioéticos na assistência perinatal: revisão integrativa da literatura
}

\author{
Bioethical conflicts in perinatal care: integrative literature review \\ Conflictos bioéticos en el cuidado perinatal: revisión integradora de la literatura
}

\section{Resumo}

Objetivo: analisar os achados disponíveis na literatura acerca dos conflitos bioéticos vivenciados pelas mulheres durante o contexto parturitivo. Métodos: Trata-se de uma pesquisa de revisão integrativa, na qual foi realizado uma busca nas seguintes bases: Medical Literature Analysis and Retrieval System Online (MEDLINE) via PubMed, Literatura Latino-Americana e do Caribe em Ciências da Saúde (LILACS) e Base de Dados em Enfermagem (BDENF), sendo as duas últimas via Biblioteca Virtual em Saúde (BVS). Utilizou-se como critério de inclusão artigos nos idiomas - português, inglês e espanhol, e recorte temporal nos últimos dez anos. Resultados: Após a busca e análise dos artigos, foram selecionados três artigos que compuseram a amostra desta pesquisa. Nestes foram identificados os conflitos bioéticos da Autonomia; Beneficência e Não-maleficência, na assistência perinatal. Conclusão: O princípio biótico que mais se apresenta em conflito nesse contexto é o da Autonomia, seguido da beneficência e não-maleficência.

Palavras-chave: Gestação; Parto; Bioética.

\begin{abstract}
Objective: to analyze the findings available in the literature about bioethical conflicts experienced by women during the birth context. Methods: This is an integrative review research, in which a search was performed in the following databases: Medical Literature Analysis and Retrieval System Online (MEDLINE) via PubMed, Latin American and Caribbean Literature on Health Sciences (LILACS) and Database in Nursing (BDENF), the last two being via the Virtual Health Library (VHL). The inclusion criteria were articles in the languages- Portuguese, English and Spanish, and time frame in the last ten years. Results: After searching and analyzing the articles, three articles were selected that made up the sample of this research. In these, the bioethical conflicts of Autonomy were identified; Beneficiency and Non-maleficence in perinatal care. Conclusion: The bioethical principle that is most in conflict in this context is that of Autonomy, followed by beneficence and non-maleficence.
\end{abstract}

Keywords: Pregnancy; Childbirth; Bioethics. 


\begin{abstract}
Resumen
Objectivo: analizar los hallazgos disponibles en la literatura sobre los conflictos bioéticos vividos por las mujeres durante el contexto del parto. Métodos Esta es una investigación de revisión integradora , en la que se realizó una búsqueda en las siguientes bases de datos: Medical Literature Analysis and Retrieval System Online (MEDLINE) vía PubMed, Latin American and Caribbean Literature on Health Sciences (LILACS) y Database in Nursing (BDENF). ), siendo los dos últimos a través de la Biblioteca Virtual en Salud (BVS). El criterio de inclusión utilizado fueron los artículos en los idiomas portugués, inglés y español, y en el marco temporal de los últimos diez años. Resultados: Luego de la búsqueda y análisis de los artículos, se seleccionaron tres artículos que conformaron la muestra de esta investigación. En estos, se identificaron los conflictos bioéticos de la Autonomía; Beneficio y No Maleficencia en la atención perinatal. Conclusión: El principio bioético más conflictivo en este contexto es el de la autonomía, seguido de la beneficencia y la no maleficencia.
\end{abstract}

Palabras clave: Embarazo; Parto; Bioética.

\title{
1. Introdução
}

Este estudo possui como objeto de pesquisa os conflitos bioéticos vivenciados pelas mulheres durante o contexto parturitivo, e surgiu a partir do cenário acadêmico das autoras na disciplina de Bioética do Mestrado em Enfermagem da Universidade Federal de Alagoas, cuja experiência possibilitou maiores reflexões acerca do tema em diversas áreas, sobretudo na pesquisa.

A Enfermagem como ciência, abrange conhecimentos científicos e técnicos, atrelados às práticas sociais, éticas e políticas vividas no ensino, na pesquisa e na assistência. Essa profissão fornece serviços ao ser humano dentro dos variados contextos que está inserida, atuando na promoção, prevenção e recuperação da saúde, através de atividades com grupos sociais ou em particular, respeitando a individualidade de cada ser (Koerich, Machado \& Costa, 2005). Podendo, com base em sua preparação profissional, atuar diretamente com a parturiente esclarecendo suas dúvidas e indicando as opções para a promoção de um parto com conforto, segurança e menos dor (Hora et al., 2021).

Vale ressaltar que, para estas ações se faz necessário recursos educacionais em saúde desde o pré-natal, envolvendo os profissionais, gestantes e familiares, facilitando a troca de saberes, para que as dúvidas sejam esclarecidas com antecedência, a fim de que as gestantes assimilem as orientações em tempo hábil (Abreu et al., 2021).

No que se refere à ética e à bioética, ambas conduzem a responsabilidade pessoal e profissional para com as questões do cotidiano e das relações humanas em todas as suas dimensões. Ética é o estudo da conduta e do caráter. Assim, atos que são considerados éticos refletem compromissos com padrões para além das preferências pessoais (Koerich, Machado \& Costa, 2005; Gerber \& Zagonel, 2013).

Nas relações entre os profissionais, ela se fundamenta mediante a responsabilidade, o compromisso com o trabalho e com o outro, bem como pelo respeito e empatia às pessoas. Desta forma, a ética se desenvolve na formação profissional frente às atitudes, valores e habilidades que são construídos no exercício dessa prática profissional (Gerber \& Zagonel, 2013).

Acerca dos cuidados com a mulher, alguns autores explicitam que, no tocante à humanização da assistência, não deve se restringir às técnicas que auxiliem no parto, mas, sobretudo, assegurar os direitos da mulher, garantir o cuidado integral, permitir que as escolhas da parturiente sejam priorizadas, para além da disponibilidade do profissional de saúde que está acompanhando, de modo que a autonomia e desejos da mulher sejam respeitados (Cunha, Gomes \& Santos, 2012).

Nesse contexto, a Bioética está inserida de modo a permitir a reflexão e direcionamento dos profissionais da saúde que estão envolvidos na assistência ao cuidado, que devem ser norteados pelos seus princípios da beneficência, não maleficência, autonomia e justiça (Santos et al., 2019).

Com base nisso, este estudo possui o objetivo de analisar os achados disponíveis na literatura acerca dos conflitos bioéticos vivenciados pelas mulheres durante o contexto parturitivo. 


\section{Metodologia}

Este estudo trata-se de uma revisão integrativa da literatura, o qual permite uma ampla abordagem metodológica, incluindo estudos experimentais e não-experimentais, com potencial para desempenhar importante papel na Prática Baseada em Evidências (PBE) na área de enfermagem (Souza et al, 2010).

O estudo desenvolveu-se em cinco fases: Formulação do problema, Coleta de dados; Avaliação dos dados; Análise e interpretação; Apresentação pública (Cooper, 1989).

Após a formulação do problema a partir da definição do objeto de pesquisa, a segunda etapa foi a coleta de dados, sendo realizada no mês de Agosto de 2021 através das bases de dados: Medical Literature Analysis and Retrieval System Online (MEDLINE) via PubMed, Literatura Latino-Americana e do Caribe em Ciências da Saúde (LILACS) e Base de Dados em Enfermagem (BDENF), sendo as duas últimas via Biblioteca Virtual em Saúde (BVS). Esta etapa ocorreu em processo de revisão aos pares, por quatros pesquisadoras, na qual cada pesquisadora realizou as etapas separadamente, com posterior comparação dos resultados encontrados.

Para a pesquisa, foram utilizados os descritores Medical Subject Headings (MeSH), "pregnant women", "bioethics" e “childbirth", por meio dos cruzamentos entre os termos com o operador booleano AND de acordo com as estratégias de busca definidas para cada uma das bases.

Os critérios de inclusão foram artigos escritos em português, inglês ou espanhol, que possuíssem estudos com relação à temática estudada, e o recorte temporal de publicações dos últimos dez anos. Os critérios de exclusão foram estudos do tipo dissertações, teses, artigos de opinião e os que estavam apenas com o resumo disponível.

Para extração dos dados da revisão, construiu-se um instrumento no Microsoft Excel ${ }^{\circledR}$ que abordava informações acerca da base de dados, descritores, resultado dos estudos, estudos excluídos, e para os estudos incluídos: autores, país, ano, título do artigo, tipo de estudo, princípios bioéticos não respeitados na assistência à mulher, conclusões e as referências.

No que diz respeito aos aspectos éticos e legais, o estudo seguiu as diretrizes da resolução do Conselho Nacional de Saúde n510/2016 e por tratar-se de uma revisão da literatura, não foi necessário o registro da pesquisa no Comitê de Ética em Pesquisa.

\section{Resultados}

Durante a coleta de dados foram encontrados um total de 43 artigos, sendo: 33 na LILACS; 06 na MEDLINE; 04 na BDENF. Após a triagem dos estudos, obteve-se uma amostra de três artigos incluídos na síntese. A Figura 1 demonstra o fluxograma de seleção amostral. 
Research, Society and Development, v. 11, n. 1, e46011125177, 2022

(CC BY 4.0) | ISSN 2525-3409 | DOI: http://dx.doi.org/10.33448/rsd-v11i1.25177

Figura 1. Fluxograma do processo seleção dos artigos nas bases de dados, 2021.

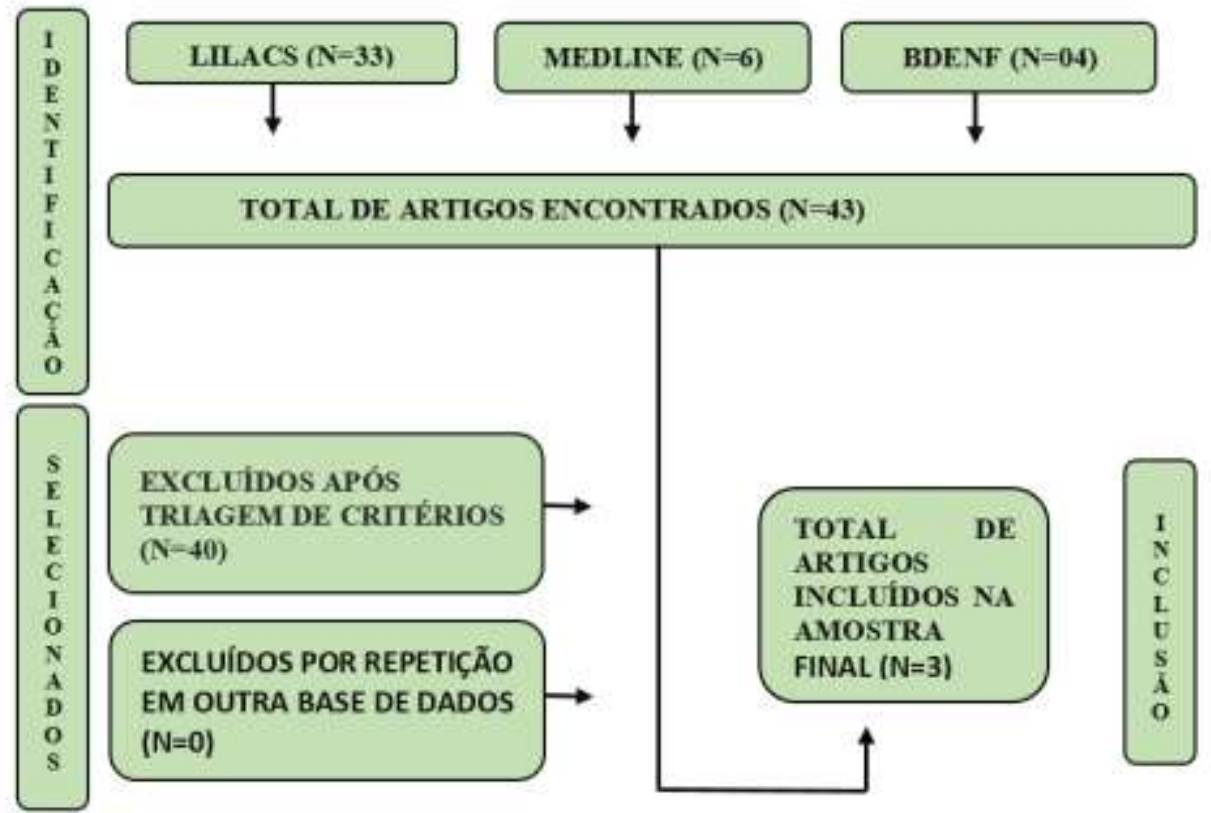

Fonte: Autores (2021).

Dos estudos selecionados, observou-se um artigo publicado no ano de 2017 e dois artigos publicados no ano de 2018. Cada artigo foi publicado em um idioma diferente, sendo eles: português, inglês e espanhol. Com relação ao tipo de estudo, cada artigo trouxe uma abordagem diferente segundo o que se observa no Quadro 1.

Quadro 1. Detalhamento dos artigos selecionados na amostra final da revisão. Brasil, 2021.

\begin{tabular}{|l|l|l|l|l|}
\hline $\begin{array}{l}\text { Identificação/ } \\
\text { Ano }\end{array}$ & Pais & $\begin{array}{l}\text { Tipo de } \\
\text { Estudo }\end{array}$ & Objetivo da pesquisa & Título \\
\hline A01 & Brasil & $\begin{array}{l}\text { Revisào } \\
\text { Integrativa }\end{array}$ & $\begin{array}{l}\text { Identificar e discutir dilemas } \\
\text { bioéticos na assistência médica a } \\
\text { gestantes adolescentes. }\end{array}$ & $\begin{array}{l}\text { Dilemas bioéticos na } \\
\text { assistència médica às } \\
\text { gestantes adolescentes }\end{array}$ \\
\hline A02 & Chile & $\begin{array}{l}\text { Estudo } \\
\text { Qualitativo }\end{array}$ & $\begin{array}{l}\text { Indagar sobre a experiência de } \\
\text { mulheres grávidas de crianças } \\
\text { incompativeis com a vida ao nascer, } \\
\text { através da história de très mulheres } \\
\text { que }\end{array}$ & $\begin{array}{l}\text { Vivencia de madres gestantes } \\
\text { de niños y niñas con } \\
\text { malformaciones } \\
\text { incompatibles con la vida }\end{array}$ \\
\hline A03 & EUA & $\begin{array}{l}\text { Revisåa } \\
\text { Narrativa }\end{array}$ & $\begin{array}{l}\text { Descrever os cuidados } \\
\text { desrespeitosos e prejudiciais, } \\
\text { tornam visivel a invisibilidade das } \\
\text { parturientes }\end{array}$ & $\begin{array}{l}\text { Obstetric Ethics and the } \\
\text { Invisible Mother }\end{array}$ \\
\hline
\end{tabular}

Fonte: Autores (2021).

No que diz respeito aos conflitos bioéticos enfrentados pelas gestantes/parturientes observou-se que os artigos trouxeram situações diversas e até polêmicas no que diz respeito à saúde reprodutiva da mulher.

Um dos artigos trouxe a reflexão e vivência de mulheres que gestaram crianças com malformações genéticas incompatíveis com a vida, discorre sobre seu processo de gestação, nascimento e puerpério, além dos profissionais envolvidos 
Research, Society and Development, v. 11, n. 1, e46011125177, 2022

(CC BY 4.0) | ISSN 2525-3409 | DOI: http://dx.doi.org/10.33448/rsd-v11i1.25177

nessa assistência, e discutem as dificuldades emocionais diante do quadro, gerando divisões e discussões acerca do aborto terapêutico, ainda ilegal no Chile, onde o estudo foi realizado (Arancibia Heger et al., 2018).

Nesse cenário, embora as mães conheçam a condição de seus filhos, existem desejos distintos entre se afastar da situação e acompanhar de perto, de modo que elas relatam sobre se sentirem protagonistas nesse momento, no sentido de fazer escolhas, inclusive no fato de expressar sua crença religiosa em batizar seu bebê.

No entanto, muitas vezes seu protagonismo se encerra ao esbarrar com outros dilemas éticos envolvendo a assistência a esse bebê, do ponto de vista institucional e médico, de modo que alguns desejos despertam nos profissionais o sentimento de distanásia, ao prolongar a vida do recém-nascido em sofrimento.

Semelhante ao estudo supracitado, em relação ao protagonismo da mulher e o desrespeito às suas escolhas e privacidade, os outros estudos retratam que, quando se trata de mães adolescentes, existem dilemas envolvendo situações de abortamento legal de forma segura e respeito ao sigilo em ética médica, e no que se refere à assistência obstétrica, deixa de ser um evento biológico, mas roteirizado pela cultura e condicionado às necessidades médicas ou institucionais, chamando atenção para a forte discussão entre a não autonomia da mãe - tida como invisível, em detrimento da beneficência do recém-nascido, ou seja, cujo objetivo seria unicamente o nascimento de um bebê saudável (De Vries, 2017; Souza et al, 2018).

Sendo assim, ao realizar uma análise dos artigos frente à bioética, percebe-se que a Autonomia se repete como principal princípio em conflito em ambos os textos, seguido pela beneficência e não-maleficência, como mostra a Tabela 2 abaixo.

Quadro 2 Síntese dos artigos quanto ao autor, ano, conclusões e princípios bioéticos em conflito.

\begin{tabular}{|l|l|l|l|}
\hline Autor & Ano & Conclusôes & Principios bioéticos em conflito \\
\hline A01 & 2018 & $\begin{array}{l}\text { Ainda há um número expressivo de gestantes } \\
\text { adolescentes no Brasil e, com isso, existem } \\
\text { conflitos envolvendo a ética médica e o aborto } \\
\text { induzido configurando-se como dilemas bioéticos, }\end{array}$ & $\begin{array}{l}\text { Autonomia, Beneficência e Não- } \\
\text { maleficência. }\end{array}$ \\
\hline A02 & 2018 & $\begin{array}{l}\text { Atualmente, é crescente o diagnóstico precoce de } \\
\text { inúmeras anomalias fetais e, no que se refere ao } \\
\text { diagnóstico de fetos incompativeis com a vida, há } \\
\text { a dificuldade emocional e dilemas éticos tanto para } \\
\text { gestantes quanto profissionais da saude. Esses } \\
\text { dilemas perpassam as complexidades do sistema } \\
\text { de saúde para um atendimento de qualidade, } \\
\text { sinalizando a necessidade de descriminalização do } \\
\text { aborto terapêutico no pais }\end{array}$ & Autonia \\
\hline A03 2017 & $\begin{array}{l}\text { A invisibilidade das màes no cenário do parto e } \\
\text { do nascimento traduz a falta de respeito aos seus } \\
\text { desejos, à sua individualidade e à autonomia, em } \\
\text { detrimento de uma ética clinica que valoriza a } \\
\text { beneficência e considera, nesse caso, o produto } \\
\text { final - bebê saudável - como o mais importante, } \\
\text { apontando um conflito matemo-fetal. }\end{array}$ & Autonomia e Beneficência \\
\hline
\end{tabular}

Fonte: Autores (2021).

\section{Discussão}

A bioética, no contexto de ciências da saúde, encontrou seu objeto nas condutas profissionais e no relacionamento profissional-paciente, de modo que é essencial essa relação para o cuidado com qualidade e segurança, interligado às questões humanísticas, de acolhimento e de assistência adequadas, sobretudo no processo parturitivo (De Barchifontaine \& Trindade, 2019; Santos et al, 2019). 
Quanto à humanização no parto, a garantia dos direitos reprodutivos da mulher se insere como um requisito importante no tocante ao respeito à sua autonomia, como princípio bioético organizador, valorizando e compartilhando seus saberes e desejos individuais (Santos et al, 2019).

Alguns autores ressaltam que a relação profissional-paciente possui potencial como prática fundamental, já que envolve dimensões técnicas, humanísticas, éticas e estéticas, estabelecendo uma relação dialética entre os atores, sobretudo ao respeitar a autonomia da mulher, cujo papel é essencial na tomada de decisão compartilhada (Sens \& Stamm, 2019).

Silva et al., em seu estudo (2021) explícita a importância do empoderamento dessa mulher desde as consultas de prénatal, visando os benefícios durante o parto e o pós-parto, como também, dar ênfase ao trabalho da equipe de enfermagem no que tange fortalecimento da autonomia da mulher sobre seus direitos e escolhas.

Em contrapartida, outro estudo abordou a relação médico-paciente como determinante para a escolha da via de parto na maioria das mulheres no Brasil, demonstrando uma supervalorização do saber técnico da equipe e desconsiderando os desejos da mulher e sua capacidade de participar do processo fisiológico do seu corpo (Rocha \& Ferreira, 2020).

Ainda segundo os autores supracitados, mesmo diante dos avanços na medicina, cujo conselho federal defende o respeito à autonomia da mulher em relação à cesárea a pedido, ainda existem falhas na comunicação desse profissional com a paciente, sendo necessária a utilização do plano de parto, que surge como ferramenta educativa para romper com o padrão autoritário e hostil dos profissionais da saúde (Rocha \& Ferreira, 2020).

No entanto, algumas ferramentas, a exemplo do plano de parto, soam aos profissionais como provocativas e abusivas, predispondo à negligência por parte da equipe, o que prejudica sumariamente o cuidado em saúde, demonstrando a fragilidade em relação ao respeito à autonomia da mulher (Sens \& Stamm, 2019).

Ao resultar em perda da autonomia e da capacidade da mulher de decidir sobre seu corpo e sexualidade, os profissionais se apropriam do corpo da mulher e seus processos reprodutivos, gerando maus tratos, medicalização e patologização do parto, o que se configura como Violência Obstétrica (Almeida \& Barbosa Ramos, 2020; Mendiri et al, 2017).

Esse tipo de violência rompe os princípios dos direitos à saúde da mulher, promovendo desigualdade entre homens e mulheres, sendo, portanto, uma manifestação da violência de gênero, havendo imposição à dor e sofrimento evitáveis (Almeida \& Barbosa Ramos, 2020; Al Adib Mendiri et al, 2017).

Quando se pensa em barreiras institucionais relativas à bioética no contexto parturitivo, os mecanismos de controle e medicalização do parto acabam sendo justificados por muitos profissionais para responder ao objetivo de terminar com o nascimento de um recém-nascido saudável, com menor morbidade materna e neonatal (Al Adib Mendiri et al, 2017).

No entanto, tratando-se do princípio da beneficência como forma de buscar o bem estar dos pacientes, o paternalismo se insere como um dos maiores problemas no cumprimento dos direitos, uma vez que a autoridade profissional não pode estabelecer uma relação de abuso, sacrificando a autonomia e desejos da paciente (Vázquez Parra, 2016).

No modelo hegemônico de assistência ao trabalho de parto e parto, há uma excessiva prática intervencionista, como a medicalização e outros procedimentos desnecessários, que potencializam a crítica relacionada ao modelo, em detrimento de boas práticas, num modelo dito como humanizado (Nicida et al, 2020)

Em um estudo realizado por Sehnem e colaboradores (2020) cita que devido às práticas intervencionistas realizadas rotineiramente, encontrou o sentimento negativo em relação ao parto, ligada ao entendimento da violência obstétrica realizada pelos profissionais de saúde, diferenciando do modelo de cuidado preconizado. Uma pesquisa destaca a importância do trabalho da equipe de enfermagem para fortalecimento das boas práticas e técnicas que preconizam o parto natural, visto que é uma maneira de dar autonomia e conforto para as mulheres em trabalho de parto e parto (Silva, Silva \& Batista, 2020).

Mesmo diante do consentimento informado, em cesarianas, alguns autores discorrem e questionam acerca do princípio da beneficência diante desse cenário, uma vez que, na maioria dos casos, a tomada de decisão é baseada apenas na 
fala ou nas informações que emanam de seus médicos, refletindo, neste caso, a autoridade do médico e não o melhor caminho para o paciente (Vázquez Parra, 2016).

\section{Conclusão}

Através da realização deste estudo pode-se concluir que, no contexto parturitivo, o princípio bioético mais infringido é o da autonomia, seguido da beneficência e não maleficência.

Dentro desse princípio, percebe-se que há um abuso por parte do profissional de saúde diante dessa relação hierárquica, no qual as escolhas da paciente não são respeitadas, ou colocadas em segundo plano, com excesso de intervenções e mecanismos de controle do parto, cuja maior preocupação é o nascimento de um recém-nascido saudável.

Sendo assim, é importante maiores reflexões e mudança de postura por parte dos profissionais, a fim de que os princípios da bioética da paciente, sobretudo a autonomia, com respeito à individualidade, desejos e privacidade da mulher, representando a garantia dos seus direitos sexuais e reprodutivos em uma assistência digna e de qualidade. Enfatizamos a importância de novos estudos sobre o tema, visando conhecer as mudanças de práticas em relação ao cuidado da mulher e a preservação dos princípios bioéticos neste processo.

\section{Referências}

Abreu, H. de SC de., Almeida, LP de., Mouta, RJO., Silva, SC de SB., Zveiter, M.., Medina, ET., Pitombeira, P., \& Santos, LL dos. (2021). Contribuição do pré-natal no preparo da gestante para o parto. Research, Society and Development, 10 (10), e405101017886. https://doi.org/10.33448/rsd-v10i10.17886.

Al Adib Mendiri, M., Ibáñez Bernáldez, M., Casado Blanco, M., \& Santos Redondo, P. (2017). La violencia obstétrica: un fenómeno vinculado a la violación de los derechos elementales de la mujer. Medicina Legal de Costa Rica, 34(1), 104-111. https://www.scielo.sa.cr/pdf/mlcr/v34n1/2215-5287-mlcr-34-01104.pdf

Arancibia Heger, M., Silva Dreyer, A., \& Pantoja Molina, F. (2018). Vivencia de madres gestantes de niños y niñas con malformaciones incompatibles con la vida. Acta bioethica, 24(1), 67-74. http://dx.doi.org/10.4067/S1726-569X2018000100067

Cooper, HM (1989). Integrando a pesquisa: um guia para revisões de literatura . Sage Publications, Inc.

Cunha, K. J. B., Gomes, L. S. V., \& Santos, R. M. A. (2012). O vivido de mulheres no parto humanizado. Revista Interdisciplinar NOVAFAPI, 5, 32-8.. <https://silo.tips/download/o-vivido-de-mulheres-no-parto-humanizado>

Barchifontaine, C. D. P., \& Trindade, M. A. (2019). Bioética, saúde e realidade brasileira.27 (3): 439-45. https://doi.org/10.1590/1983-80422019273327.

De Vries, R. (2017). Obstetric ethics and the invisible mother. Narrative inquiry in bioethics, 7(3), 215-220.https://doi.org/10.1353/nib.2017.0068.

Almeida, N. M. D. O., \& Ramos, E. M. B. (2020). O direito da parturiente ao acompanhante como instrumento de prevenção à violência obstétrica. Cadernos Ibero-Americanos de Direito Sanitário, 9(4), 12-27. https://doi.org/10.17566/ciads.v9i4.643

Gerber, V. K. D. Q., \& Zagonel, I. P. S. (2013). A ética no ensino superior na área da saúde: uma revisão integrativa. Revista Bioética, 21, 168-178. https://www.scielo.br/j/bioet/a/Wx4nqzqcyWQPPMSFF4dtNjb/?lang=pt\&format=pdf

Hora, A. B. ., Santos, A. K. S. ., Oliveira, C. S. de ., Feitosa, M. C. G. de O. ., Teles, W. de S. ., Silva, M. C. da ., Calasans, T. A. S. ., Barros, Ângela M. M. S. ., Torres, R. C. ., Azevedo, M. V. C. ., Andrade, A. F. S. M. de ., Santos Junior, P. C. C. ., Debbo, A., \& Silva, M. H. S. . (2021). The importance of the nurse's role in the humanization of childbirth: comprehensive verification. Research, Society and Development, 10(13), e266101321253. https://doi.org/10.33448/rsdv10i13.21253

JC, V. P. Abuso de la operación cesárea y el principio de beneficencia. Rev Latinoam Bioet [Internet]. 2016 [cited 2016 Aug 24]; 16 (1): $60-71$.

Koerich, M. S., Machado, R. R., \& Costa, E. (2005). Ética e bioética: para dar início à reflexão. Texto \& Contexto-Enfermagem, 14, 106110.<https://doi.org/10.1590/S0104-07072005000100014>

Nicida, L. R. D. A., Teixeira, L. A. D. S., Rodrigues, A. P., \& Bonan, C. (2020). Medicalização do parto: os sentidos atribuídos pela literatura de assistência ao parto no Brasil. Ciência \& Saúde Coletiva, 25, 4531-4546. https://doi.org/10.1590/1413-812320202511.00752019

Rocha, N. F. F. D., \& Ferreira, J. (2020). A escolha da via de parto e a autonomia das mulheres no Brasil: uma revisão integrativa. Saúde em Debate, 44, 556568.https://doi.org/10.1590/0103-1104202012521

Santos, A.L.S. et al. (2019). Bioética e parto humanizado: concepções e práticas de enfermeiras obstétricas. Revista Textura, v. 13, n. 21, p. 093-103. DOI: https://doi.org/10.22479/desenreg2019v13n21p93-103 
Research, Society and Development, v. 11, n. 1, e46011125177, 2022

(CC BY 4.0) | ISSN 2525-3409 | DOI: http://dx.doi.org/10.33448/rsd-v11i1.25177

Sehnem, GD, Rios, CPP, Souza, MB de, Arboit, J., Cogo, SB, Mutti, CF, Scopel, MF, \& Birrer, JA (2020). Intervenções obstétric as durante o processo de parturição: percepção da puérpera. Pesquisa, Sociedade e Desenvolvimento , 9 (6), e131963515. https://doi.org/10.33448/rsd-v9i6.3515

Sens, M. M., \& Stamm, A. M. N. D. F. (2019). Percepção dos médicos sobre a violência obstétrica na sutil dimensão da relação humana e médico-paciente. Interface-Comunicação, Saúde, Educação, 23. https://doi.org/10.1590/Interface.180487.

Silva, A. F. P. M. da.; Almeida, B. G. R. F. de .; Ribeiro, E. A.; Teixeira, L. C.; Silva, P. C. P. de O. da .; Ribeiro, A. da S. (2021). Violência obstétrica relacionada a perda de autonomia da mulher na sala de parto. Research, Society and Development, v. 10, n. 5, p. e22210514814. https://doi.org/10.33448/rsdv10i5.14814

Souza, E. V. D., Silva, V. S. B. D., Lozado, Y. A., Bomfim, E. D. S., Alves, J. P., Boery, E. N., \& Boery, R. N. S. D. O. (2018). Dilemas bioéticos na assistência médica às gestantes adolescentes. Revista bioética, 26, 87-94. https://doi.org/10.1590/1983-80422018261229

Souza, M. T. D., Silva, M. D. D., \& Carvalho, R. D. (2010). Revisão integrativa: o que é e como fazer. Einstein (São Paulo), 8, 102-106 https://doi.org/10.1590/S1679-45082010RW1134 